\title{
CoEnzyme Q10: A new horizon in the treatment of periodontal diseases
}

\author{
AKMS Kadir ${ }^{1, *}$, AA Rabbi ${ }^{2}$, MM Rahman ${ }^{3}$ \\ ${ }^{\mathbf{1}, 2}$ Institute of Biological Sciences, Rajshahi University, Bangladesh, ${ }^{3}$ National Institute of Preventive \& Social Medicine, Dhaka, \\ Bangladesh
}

*Corresponding Author:

Email: dentist.kadir@gmail.com

\begin{abstract}
Periodontitis is an inflammatory process, initiated by the plaque biofilm that leads to loss of periodontal attachment to the root surface and adjacent alveolar bone and gradually results in tooth loss. Periodontal pathogens can induce reactive oxygen species overproduction and thus may cause collagen and periodontal cell breakdown. Reactive oxygen species and free radicals are thought to be involved in the pathogenesis of a variety of inflammatory disorders by increasing oxidative stress at the tissue and cellular level. Oxidative stress arises within tissues when the normal balance between reactive oxygen species generation and antioxidant defense is altered and excess of reactive oxygen species and/or a depletion of antioxidants occur. When reactive oxygen species are scavenged by antioxidants, there can be a reduction of collagen degradation. Various forms of antioxidants have been introduced as an approach to fight dental diseases and improve general gingival health. Coenzyme Q10 serves as an intercellular antioxidant and its concentration is increased in the diseased gingiva which effectively suppresses advanced periodontal inflammation. This article focuses on the effect of Coenzyme Q10 on treating periodontal disease.
\end{abstract}

Keywords: Free Radicals, Reactive Oxygen Species, Antioxidant, Periodontal Diseases, Coenzyme Q10.

\section{Introduction}

Periodontal disease is a chronic bacterial infection that affects the gingivae and other tissues that support the teeth. Gingivitis, the most common type, affects the gum tissue solely and is caused by the accumulation of bacterial plaque, leading to a chronic inflammatory process. Red, swollen, tender, bleeding gums, receding gums, pockets separating the gums from the teeth, and bad breath are all possible manifestations of gingivitis. ${ }^{(1)}$ If not properly treated, gingivitis can progress to periodontitis, and the inflammatory process may spread to periodontal ligaments and alveolar bone. If these structures were eventually destroyed, teeth would loosen and fallout. ${ }^{(2)}$ Primary causative agents for gingival and periodontal diseases have been shown to be predominantly gram negative anaerobic or facultative anaerobic bacteria within the sub gingival biofilm. ${ }^{(3)}$ Exaggerated host response to those organisms and their products cause the majority of periodontal tissue destruction. ${ }^{(4)}$ The neutrophils are the first line of defense against this infectious periodontal disease and play an important role in host defense by controlling bacterial invasion through intracellular and extracellular oxidative and non-oxidative killing mechanisms. ${ }^{(5)}$ Reactive oxygen species (ROS) are formed during oxidative killing mechanism of neutrophils and other phagocytes. ${ }^{(6)}$ In recent years, hydrogen peroxide $\left(\mathrm{H}_{2} \mathrm{O}_{2}\right)$, hypochlorous acid $(\mathrm{HOCl})$, ozone $\left(\mathrm{O}_{3}\right)$ and singlet oxygen $\left({ }^{1} \mathrm{O}_{2}\right)$ are included as "reactive oxygen species" (ROS) which are non-radical in nature and capable of radical transformation in the extra and intracellular environments. ${ }^{(7)}$ Free radicals are highly reactive and diverse in nature, can extract electrons and oxidize a number of bio molecules which play a vital role in cell and tissue function. ${ }^{(5)}$ Besides their extremely short half-lives, most ROS can initiate free radical chain reactions causing substantial tissue damage. Anti-Oxidant (AO) mechanisms of the body specifically remove the formed harmful oxidants (ROS), or repair the damage done by ROS. ${ }^{(7)}$ Physiologically, there is a meaningful balance between ROS activity and antioxidant defense capacity. Oxidative stress results if the balance is lost because of the reduction in anti-oxidant defenses or an increase in ROS production or activity. This imbalance between the Reactive Oxygen Species and Anti-Oxidant has been marked as one of the progressive or pathogenic factors for periodontal disease.

Coenzyme Q10 (also known as Coenzyme Q, CoQ, CoQ10, Ubiquinone, Ubiquinone-Q10, Ubidecarenone, and Vitamin Q10) is a compound found naturally in the mitochondria. CoQ10 is also known as ubiquinone because of its ubiquitous presence in the nature and its quinine structure (similar to that of vitamin $\mathrm{K}$ ). ${ }^{(8,9)} \mathrm{A}$ cell's major energy source is adenosine triphosphate (ATP) which drives a number of biological processes and that includes muscle contraction and the production of protein. The biosynthesis of ATP involves a series of redox reactions where CoQ10 acts as a cofactor in the electron transport chain. CoQ10 also works as an antioxidant. ${ }^{(8,9)}$ Antioxidants are substances that scavenge free radicals, damaging compounds in the body that alter cell membranes, tamper with DNA, and even cause cell death. Antioxidants like CoQ10 can neutralize free radicals and may reduce or even help prevent some of the damage caused by free radicals. ${ }^{(10,11)}$ Bodies can synthesis CoQ10, but sometimes body's synthetic capacity is not enough to fulfill CoQ10 requirements. Metabolically active cells (such as heart, immune system, gingiva and gastric 
mucosa) are susceptible to CoQ10 deficiency as they require a great amount of CoQ10 for normal function. ${ }^{(10)}$ In medical and dental conditions like cardiovascular diseases, periodontal diseases, gastric ulcer, cancer and acquired immunodeficiency syndrome (AIDS), tissue deficiencies of CoQ10 occur. A deficiency may result either from impaired synthesis due to nutritional deficiencies, or, CoQ10 levels decline with advancing age, or, genetic or acquired defect in synthesis or utilization, or, increased tissue needs resulting from illness. ${ }^{(12)}$

\section{Historical Background}

In 1955, Festenstein et al. ${ }^{(13)}$ the scientists in Morton's Laboratory in Liverpool (England) isolated an unsaponifiable lipid with a striking ultraviolet absorption at $272 \mathrm{~nm}$ from the intestinal mucosa of horses. Morton named it ubiquinone because the new substance was identified as a quinone and was found to be widely distributed in animal tissues, (ubiquitous quinone - everywhere present quinone). Two years later in 1957, Crane et al. ${ }^{(14)}$ observed a novel quinone in the lipid extracts of mitochondria and named it coenzyme $\mathrm{Q}$ because of its participation in the electron transport chain in David Green's Laboratory at the University of Wisconsin (USA) and in 1958, its chemical structure was reported by Dr. Karl Folkers and coworkers at Merck. In 1961, Peter Mitchell ${ }^{(15)}$ proposed the electron transport chain (which includes the vital proton-motive role of CoQ10). He received a Nobel Prize for this invention in 1978. In 1972, Gian Paolo Littarru ${ }^{(16)}$ and Karl Folkers ${ }^{(16)}$ separately demonstrated a deficiency of CoQ10 in human heart disease. The antioxidant role of the molecule as a free radical scavenger was widely studied by Lars Ernster et al. ${ }^{(8)}$ CoQ10 has become a science subject of many researches.

\section{Chemical and Physical Properties of Coenzyme Q10}

Ubiquinone, chemically 2,3-dimethoxy-5-methyl6-polyisoprene parabenzoquinone(Fig. 1), is an orange lipophilic, odourless and tasteless powder in its natural form.<smiles>CCCCCC=CCC1=C(C)C(=O)C(OC)=C(OC)C1=O</smiles>

Fig.1: Chemical structure of coenzyme Q10 (2,3dimethoxy-5-methyl-6-ten-isoprene parabenzoquinone)

Because CoQ10 has 10 isoprenoid units hence its name is coenzyme Q10. Molecular weight of CoQ10 is
$863.34 \mathrm{~g} / \mathrm{mol}$. CoQ10 is very unstable and deteriorates at about $46^{\circ} \mathrm{C} .{ }^{(17)}$ It has a similarity with the structure of vitamin $\mathrm{K}$ and vitamin $\mathrm{E}$ that comprises a benzoquinone ring with an isoprenoid side chain and is a biologically active quinone. CoQ10 is the most extensive form in humans and most mammals and has also been found in other animals, microorganisms, and plants. CoQ9 is the primary form found in rats and mice. ${ }^{(18)}$ The main chemical characteristic of CoQ10is its existence in three alternate redox states which is responsible for its various functions $\mathbf{s}^{(8,19,20)}$ and they are:

1. The fully oxidized ubiquinone form which converts first into a partially reduced form, upon two sequential additions of hydrogen atoms,

2. Semiquinone - free radical form;

3. The fully reduced ubiquinol form (Fig. 2).

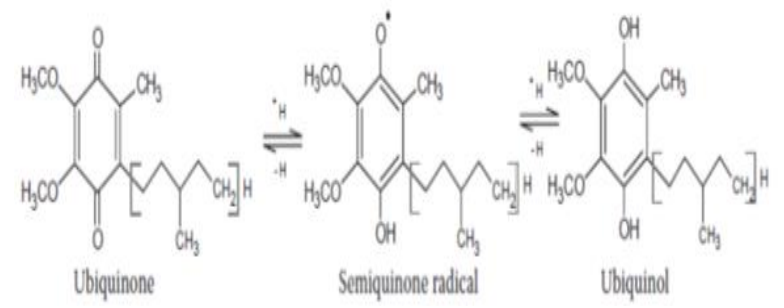

Fig.2: Three Redox states of coenzyme Q10

CoQ10 can exist in either the cis. or the trans. forms but only the trans. form is found in nature. Yet, both forms can be produced in a mixture by means of bio-fermentation or a chemical process. ${ }^{(21)}$

\section{Biochemistry in the Human}

In human organism, normal CoQ10 level in blood is $0.7-1 \mu \mathrm{g} / \mathrm{ml} .{ }^{(22)}$ The biosynthesis of CoQ10 from the amino acid tyrosine is a 17-step process (Fig. 3) which requires at least eight vitamins (riboflavin, pyridoxine, niacinamide, ascorbic acid, cobalamin, folic acid, tocopherol, and pantothenic acid) and several trace elements. ${ }^{(23,24)}$ After oral administration, CoQ10 is slowly absorbed and is taken up by chylomicron, distributed to the liver and incorporated into very low density lipoproteins. ${ }^{(25,26)} 5-10$ hours after ingestion peak blood levels occur and the elimination half life is 34 hours. CoQ10 is primarily excreted through the biliary tract. Normal serum levels become double when typical adult daily doses is 100 - 150 milligrams. ${ }^{(26)}$ Depending on the physiochemical characters in the preparation of coenzyme Q10from powder, suspension, oil solution, or solubilized form, absorption of the substance varies and bioavailability differs. In a Study, solubilized coenzyme Q10 has shown better absorption, higher plasma concentration, and consequently better bioavailability which indicates that plasma concentrations of coenzyme Q10 are 2-2.5 times higher during long-term oral therapy with solubilized forms and the bioavailability is 3-6 times higher comparing with powder. ${ }^{(27-29)}$ 


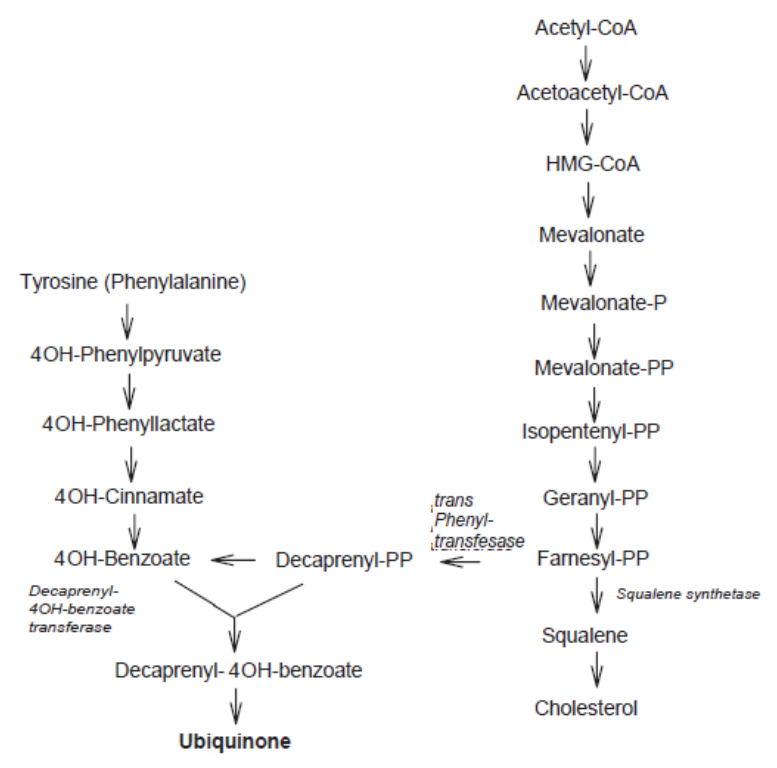

Fig. 3: Biosynthesis of ubiquinone from tyrosine

\section{Role of Coenzyme Q10}

CoQ10 has four important roles physiologically: ${ }^{(26,30)}$

1. It has an essential role in mitochondrial energy (ATP) production through redox activity in the respiratory chain, transporting electrons between enzymes.

2. In the cell membrane and endo membranes, extra mitochondrial redox activity is played by CoQ10.

3. It plays an important role in membrane stabilization and fluidity.

4. CoQ10 inhibits lipid peroxidation and scavenges free radicals, thus functions as an antioxidant.

\section{Antioxidant Function of Coenzyme Q10}

The energy carrier function of CoQ10 gives rise to the antioxidant characteristics. The CoQ10 molecule is continuously going through an oxidation-reduction cycle; hence it acts as an energy carrier. By accepting electrons, it becomes reduced and by it giving up electrons, it becomes oxidized. The CoQ10 molecule holds electrons rather loosely in its reduced form, so this CoQ molecule will quite easily give up one or both electrons and, thus, act as an antioxidant. By preventing the production of lipid peroxyl radicals (LOO), (Fig. 4) CoQ10 inhibits lipid peroxidation. ${ }^{(31)}$ Moreover, the reduced form of $\mathrm{CoQ}$ effectively regenerates vitamin $\mathrm{E}$ from the alpha-tocopheroxyl radical and, thereby interfering with the generation step. Furthermore, interaction of $\mathrm{H}_{2} \mathrm{O}_{2}$ with metal ions bound to DNA generates hydroxyl radicals during oxidative stress, and CoQ efficiently prevents the oxidation of bases, particularly, in mitochondrial DNA. Differing from other antioxidants, this compound inhibits both the initiation and the increase in the lipid and protein oxidation. ${ }^{(32)}$

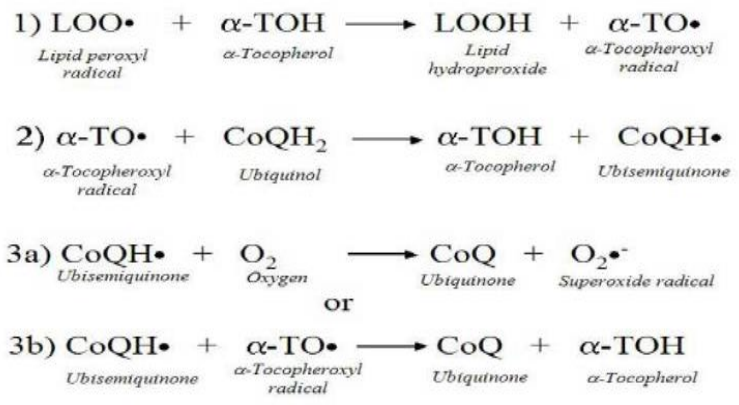

Fig. 4: Reaction scheme of Lipid peroxyl radical with alpha-Tocopherol

\section{Effect of Coenzyme Q10 in Periodontitis}

Accumulation of subgingival plaque gradually causes chronic periodontitis. As the plaque contains extremely complex microflora, determination of organisms responsible for tissue destruction associated with the disease is really problematic. Investigators found that the subgingival flora of healthy gingival crevice is slender and consists of aerobic and facultative bacteria in greater proportion. In diseased condition, anaerobic bacteria increase in ratio. Tissue destruction caused directly by toxic products of these bacteria and indirectly by inflammatory process which activates host defense systems are observed. ${ }^{(33)}$

Mammalian inflammation like event characterized by tissue infiltration by monocytes and polymorphonuclear leukocytes (PMN L) and features following phagocytosis like non-mitochondrial $\mathrm{O}_{2}$ consumption, which may be 10 or 20 times that of resting consumption. Ultimately free radicals (FRs) and reactive oxygen species (ROS), such as superoxide anion radicals, hydrogen peroxide, hydroxyl radicals, and hypochlorous acid, all capable of damaging either cell membranes or associated biomolecules are generated. ${ }^{(33)}$ Several FRs and ROS can rapidly modify either small, free biomolecules (i.e., vitamins, amino acids, carbohydrates, and lipids) or macromolecules (i.e., proteins, nucleic acids) or even supramolecular structure (i.e., cell membranes, circulating lipoproteins) because of their high reactivity. The type and the extent of damage depend upon the site of generation. Usually, the anti-oxidant defense mechanisms of the surrounding tissues can perfectly control the oxidative damage but plaque microorganisms promoting periodontitis can create imbalance in this equilibrium. A phenomenon is produced in which a massive neutrophil migration to the gingiva and gingival fluid takes place leading to abnormal spreading of FR/ROS. For this reason, a search for appropriate "antioxidant therapy" in inflammatory periodontal disease was mandatory. ${ }^{(34)}$

Coenzyme Q10 could be deficient at its enzyme sites in gingival tissue because of periodontal disease 
and/ or may exist independently. If nutritional deficiency of coenzyme Q10 existed in gingival tissue without periodontal disease, then the manifestation of periodontal disease could promote the gingival deficiency of coenzyme Q10. ${ }^{(35)}$ Oral and dental treatment and oral hygiene in such patients could correct the plaque and calculus, but the CoQ10 deficiency could not be corrected due to systemic cause. CoQ10 therapy along with the oral hygiene can improve this type of periodontal disease. ${ }^{(35)}$

The specific activity of succinic dehydrogenasecoenzyme Q10 reductase in gingival tissues from patients with periodontal disease against normal periodontal tissues has been evaluated using biopsies. It showed a deficiency of CoQ10 in patients with periodontal disease. An increase in the specific activity of this mitochondrial enzyme was found in deficient patients on exogenous CoQ10 administration. ${ }^{(35)}$ The periodontal score was also decreased concluding that CoQ10 should be considered as an adjunct for the treatment of periodontitis in current dental practice. ${ }^{(36)}$

International English language dental literature did not have firm documentation on the effect of coenzyme Q10 on periodontal disease. The review of available literature does not give any foundation for the claims indicating the benefits of coenzyme Q10 and has no place in periodontal treatment. ${ }^{(37)} \mathrm{A}$ study evaluated the periodontium condition after oral applications of coenzyme Q10 with vitamin E. The total antioxidant status (TAS) in the mixed saliva by the colorimetric method was determined twice. The average value of plaque index decreased from 1.0 to 0.36 , average value of interdental hygiene index was reduced from 39.51$6.97 \%$, gingival index values decreased from 0.68 to 0.18 , and the values of sulcus bleeding index decreased from 7.26 to 0.87 . Periodontal pockets also lowered by $30 \%$. The laboratory examination result improved by $20 \%$. It concluded that coenzyme Q10 with vitamin E had a beneficial effect on the periodontal tissue. ${ }^{(38)}$

Coenzyme Q10 as an antioxidant has received much research attention in the medical literature in the last several years. Besides of the thought as an alternative medication, many dentists routinely use Coenzyme Q10 both topically and systemically.

Mode of application of Coenzyme Q10 in periodontitis patients

Coenzyme Q10 can be applied topically, subgingivally or, intrapocket application can also be done. ${ }^{(39)}$

1. Topical application: With the tip of the applicator which is completely soaked in gel and applied to the assigned quadrant. (Fig. 5)

2. Intrapocket application: With the help of irrigation needles to deliver the gel in the intrapocket. (Fig. 6)

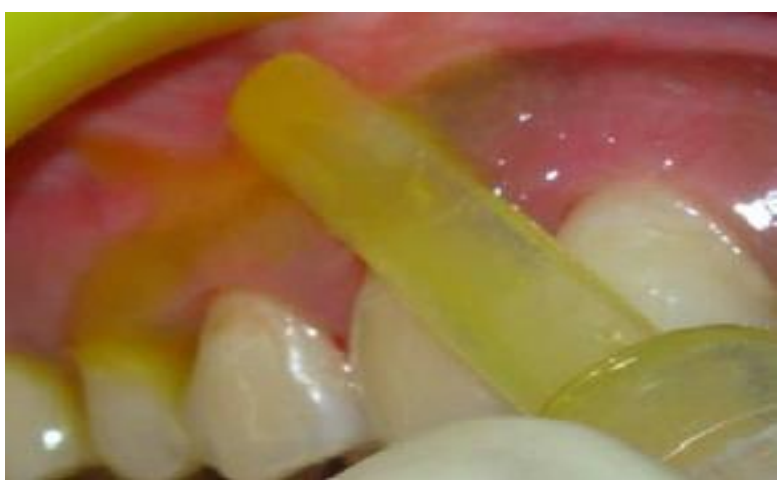

Fig. 5: Topical Application of Coenzyme Q10

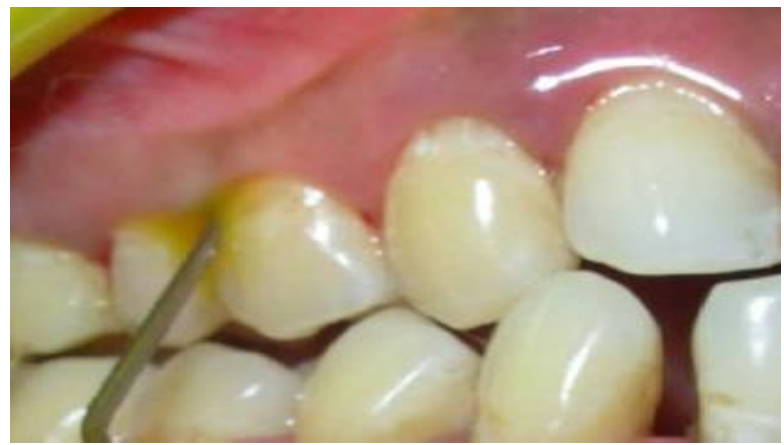

Fig. 6: Intrapocket Application of Coenzyme Q10

(Courtesy: Dr. Humera Parvez, Department of Periodontology, Rungta College of Dental Sciences and Research, Bhilai, Chhattisgarh, India. A comparative evaluation of topical and intrasulcular application of coenzyme Q10 (Perio $\mathrm{Q}^{\mathrm{TM}}$ ) gel in chronic periodontitis patients: A clinical study. Journal of Indian Society of Periodontology, Vol. 18, No. 4, July-August, 2014, pp. 461-465)

3. Subgingival administration: By inserting the syringe first to the base of the periodontal pocket and then working the way up until the gingival margin while placing the gel. (Fig. 7)

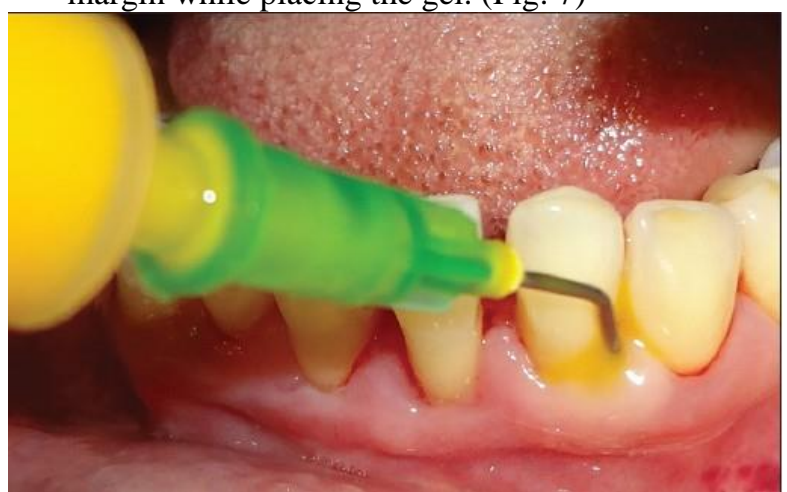

Fig. 7: Sugingival Administration of Coenzyme Q10 (Courtesy: Dr. Humera Parvez, Department of Periodontology, Rungta College of Dental Sciences and Research, Bhilai, Chhattisgarh, India. A comparative evaluation of topical and intrasulcular application of coenzyme Q10 (Perio $\mathrm{Q}^{\mathrm{TM}}$ ) gel in chronic periodontitis 
patients: A clinical study. Journal of Indian Society of Periodontology, Vol. 18, No. 4, July-August, 2014, pp. 461-465.)

\section{Side Effects of Coenzyme Q10}

CoQ10 treatment is safe, even at very high doses. Most of the studies have not reported significant side effects of CoQ10 therapy. The most experienced side effects reported by the patients using CoQ10 include mild insomnia, elevated level of liver enzymes, rashes, nausea, vomiting, abdominal discomfort, diarrhea. ${ }^{(8)}$

\section{Conclusion}

The concept of ROS-induced destruction has led to search for an appropriate complimentary antioxidant therapy in the treatment of numerous diseases including inflammatory periodontal diseases. Coenzyme Q10 is an endogenous lipid-soluble antioxidant found in all organisms. The exciting new findings by scientific researchers suggest that $\mathrm{CoQ} 10$ is an important antioxidant readily used by the body and delaying various progresses of diseases. Because it is an antioxidant, there is not sufficient new information for coenzyme Q10 in the treatment of periodontal conditions. The pharmacology of coenzyme Q10 indicates that it may be an agent for treatment of periodontitis. Additional studies are needed to define its precise role, appropriate dose, effectiveness, and bioavailability in the treatment of periodontitis, not only as an adjunct but also as a major therapeutic agent.

\section{Acknowledgement}

We would like to extend our gratitude to Dr. Surekha Rathod, Professor, Department of Periodontics, VSPM Dental College and Research Centre, Nagpur, India and her co-authors; Dr. Sweta Soni, Senior Lecturer, Department of Periodontics, Vyas Dental College and Hospital, Jodhpur, India and her coauthors; from whom we got our inspiration to write this review article.

\section{References}

1. The Canadian Dental Association. Your oral health . http://www.cda adc.ca/en/oral health/index.

2. Gaby, A. Nutritional Medicine. Concord, NH: Frits Perlberg Publishing, 2011, 1374 pages.

3. Haffajee AD, Socransky SS. Microbial etiological agents of destructive periodontal diseases. Periodontology 2000. 1994;5:78-111.

4. Lamster IB, Novak MJ. Host mediators in gingival crevicular fluid: implications for the pathogenesis of periodontal disease. Critical Reviews in Oral Biology and Medicine.1992;3(1):31-60.

5. Dennison DK, Dyke TE. The acute inflammatory response and the role of phagocytic cells in periodontal health and disease. Periodontology 2000. 1997;14(1):5478.

6. Roos D, van Bruggen R, Meischl C. Oxidative killing of microbes by neutrophils. Microbes and Infection. 2003;5(14):1307-15.
7. Waddington R, Moseley R, Embery G. Periodontal Disease Mechanisms: Reactive oxygen species: a potential role in the pathogenesis of periodontal diseases. Oral Diseases. 2000;6(3):138-51.

8. Ernster, L; Dallner, G (1995)."Biochemical, physiological and medical aspects of ubiquinone function". Biochimica Biophysica Acta (1):195-204.

9. Al-Hasso. Coenzyme Q10: a review. Hosp Pharm. 2001;36(1):51-66.

10. Raitakari OT, McCredie RJ, Witting P, Griffiths KA, Letter J, Sullivan D, Stocker R, Cele rmajer DS. Coenzyme Q improves LDL resistance to ex vivo oxidation but does not enhance endothelial function in hyper cholesterolemic young adults. Free Radic Biol Med.2000;28(7):1100-1105.

11. Aberg, F; Appelkvist, EL; Dallner, G; Ernster, L (1992). "Distribution and redox state of ubiquinones in rat and human tissues". Archives of biochemistry and biophysics 295(2):230-4.

12. Shindo, Y; Witt, E; Han, D; Epstein, W; Packer, L (1994). "Enzymic and non-enzymic antioxidants in epidermis and dermis of human skin". The Journal of investigative dermatology 102(1):122-4.

13. Festenstein G.N., Heaton F.W., Lowe J.S., Morton R.A. (1955): A constituent of the unsaponifiable portion of animal tissue lipids. Biochemical Journal, 59:558-566.

14. Crane F.L., Hatefi Y., Lester R.L., Widmer C. (1957): Isolation of a quinone from beef heart and beef heart mitochondria. Biochimica et Biophysica Acta, 25:220 221.

15. Mitchell M. (1961): Coupling of phosphorylation to electron and hydrogen transfer by a chemiosmotic type of mechanism. Nature, 191:144-148.

16. Littarru G.P., Ho L., Folkers K. (1972): Deficiency of coenzyme Q10 in human heart disease. Part I and II. International Journal for Vitamin and Nutrition Research, 42/2:291,42/3:413.

17. US Patent (2005): Formulation and manufacturing process for coenzyme Q10 soft gel capsules. US patent $6,855,733$.

18. Ramasarma T. (1985): Natural Occurrence and Distribution of Coenzyme Q. In: Lenaz G. (ed.): Coenzyme Q. Biochemistry, Biogenetics and Clinical Applications of Ubiquinone. John Wiley and Sons, New York: 67-81.

19. Battino M., Ferri E., Gorini A., Villa F.R., Huertas R.J.F., Fiorella P., Genova M.L., Lenaz G., Marchetti M. (1990): Natural distribution and occurrence of coenzyme Q homologues. Membrane Biochemistry, 9: 179-190.

20. James A.M., Smith R.A.J., Murphy M.P. (2004): Antioxidant and prooxidant properties of mitochondrial coenzyme CoQ10. Archives of Biochemistry and Biophysics, 423:47-56.

21. US Patent (2003): Synthesis of coenzyme Q10 ubiquinone. US patent 6,506,915.

22. Lockwood K, Moesgaard S, Hanioka T, Folkers K. Apparent partial remission of breast cancer in 'high risk' patients supplemented with nutritional antioxidants, essential fatty acids and coenzyme Q10. Mol Aspects Med 1994;15: s231-40.

23. Hojerova J. (2000): Koenzym Q10 - vyznam, vlastnosti a využitie vo vyžive a kozmetike. Česka a slovenska farmacie, 49:119-123.

24. Crane F.L. (2001): Biochemical functions of coenzyme Q10. Journal of the American College of Nutrition, 20:591-598.

25. Folkers K. Relevance of the biosynthesis of coenzyme Q10 and of the four bases of DNA as a rationale for the 
molecular causes of cancer and a therapy. Biochem Biophys Res Commun 1996;224:358-61.

26. Greenberg S, Frishman WH. Coenzyme Q10: a new drug for cardiovascular disease. J Clin Pharmacol1990;30:596608.

27. Kalenikova EI, Gorodetskaya EA, Kolokolchikova EG, Shashurin DA, Medvedev OS. Chronic administration of coenzyme Q10 limits post infarct myocardial remodeling in rats. Biochemistry (Mosc) 2007;72:407-15.

28. Chopra RK, Goldman R, Sinatra ST, Bhagavan HN. Relative bioavailability of coenzyme Q10 formulations in human subjects. Int J Vitam Nutr Res 1998;68:109-13.

29. Miles M, Horn P, Miles L, Tang P, Steele P, DeGraw T. Bioequivalence of coenzyme Q10 from over-the-counter supplements. Nutr Res 2002;22:919-29.

30. Rauchova H, Drahota Z, Lenaz G. Function of coenzyme $\mathrm{Q}$ in the cell: some biochemical and physiological properties. Physiol Res1995;44:209-16.

31. Bentinger M, Tekle M, Dallner G:Coenzyme Q-biosynthesis andfunctions. Biochem Biophys Res Commun 2010,396(1):74-79.

32. Tomasetti M, Alleva R, Borghi B, Collins AR. Collins In vivosupplementation with coenzyme Q10enhances the recovery of human lymphocytes from oxidative DNA damage. FASEB J. 2001;15:1425-7.

33. Miyake Y., Shouzu A., Nishikawa M., Yonemoto T., Shimizu H., Omoto S., Hayakawa T., Inada M. (1999): Effect of treatment of 3-hydroxy-3-methylglutaryl coenzyme A reductase inhibitors on serum coenzyme Q10 in diabetic patients. Arzneimittel-Forschung, 49:324-329.

34. Battino M, Bullon P, Wilson M, Newman H. Newman Oxidative injury and inflammatory periodontal diseases: The challenge of anti-oxidants to free radicals and reactive oxygen species. Crit Rev Oral Biol Med. 1999;10:458-476.

35. Nakamura R, Littarru GP, Folkers K, Wilkinson EG. Deficiency of coenzyme $Q$ in gingival tissue from patients with periodontal disease. Int J Vitam Nutr Res. 1973;43:84-92.

36. Wilkinson EG, Arnold RM, Folkers K. Bioenergetics in clinical medicine. VI. Adjunctive treatment of periodontal disease with CoQ10. Res Commun Chem Path Pharmac. 1976;14:715-719.

37. Watts TL. Coenzyme Q10 and periodontal treatment: Is there any beneficial effect? Br Dent J.1995;178:209-213.

38. Brzozowska TM, Flisykowska AK, OEwitkowska MW, Stopa J. Healing of periodontal tissue assisted by Coenzyme Q10 with Vitamin E: Clinical and laboratory evaluation. Pharmacol Rep. 2007;59:257-260.

39. Srinivasa Tenka Sale, Humera Parvez, Ramreddy Yeltiwar, Gopinath Vivekanandan, Aena Jain Pundir, Priya Jain. A comparative evaluation of topical and intrasulcular application of coenzyme Q10 (Perio Q ${ }^{\mathrm{TM}}$ ) gel in chronic periodontitis patients: A clinical study. Journal of Indian Society of Periodontology, Vol. 18, No. 4, July-August, 2014, pp. 461-465. 